\title{
Antibiofilm potential of Lactobacillus plantarum spp. cell free supernatant (CFS) against multidrug resistant bacterial pathogens
}

\author{
Hojjatolah Zamani ${ }^{1 *}$, Saeid Rahbar ${ }^{2}$, Seyed Reza Garakoui ${ }^{3}$, Anahita Afsah Sahebi ${ }^{1}$, Hannaneh Jafari ${ }^{1}$ \\ ${ }^{1}$ Department of Biology, Faculty of Science, University of Guilan, Rasht, Iran \\ ${ }^{2}$ Young Researchers and Elites Club, Rasht Branch, Islamic Azad University, Rasht, Iran \\ ${ }^{3}$ Section of Microbiology, Sobhan Oncology Pharmaceutical Company, Rasht, Iran
}

Received: Aug 8, 2017, Revised: Nov 13, 2017, Accepted: Nov 28, 2017

\begin{abstract}
Biofilm formation is a major determinant factor in development of bacterial infections. In addition, bacteria embedded in a biofilm are more resistant to antimicrobials and thus the ability of bacteria to persist and grow in a biofilm seems to be the major factor for pathogenesis and therapeutic failure. In the current study, a Lactobacillus plantarum spp was isolated from Siahmazgi cheese, traditional cheese of Guilan province, Iran, and was identified using morphological, biochemical and molecular identification assays. Antibiofilm potential of the Lactobacillus plantarum spp cell free supernatant (CFS) against multidrug resistance Pseudomonas aeruginosa, Staphylococcus aureus and Escherichia coli was characterized. According to the results, the CFS not only reduced biofilm formation by pathogenic bacteria, but also disrupted preformed biofilms. The CFS remained unaffected by chemicals including EDTA, SDS and Tween 80 , and showed stability at high temperatures $\left(80\right.$ and $\left.100{ }^{\circ} \mathrm{C}\right)$, as well as a wide range of $\mathrm{pH}$. However, the antibiofilm activity was inhibited after treating with proteinase $\mathrm{K}$. According to these results, L. plantarum spp could be regarded as a suitable strain to produce antibiofilm agents which could be used for preventive and therapeutic approaches.
\end{abstract}

Keywords: Antibiofilm, multidrug resistance, lactic acid bacteria, probiotics

Pharm Biomed Res 2017; 3(2): 39-44

\section{Introduction}

Traditional dairy products have been considered as good sources of lactic acid bacteria (LAB) and ideal vehicles to deliver beneficial bacteria to human gastrointestinal tract $\mathrm{LAB}$ are good candidates to be used as probiotics due to their non-pathogenic nature, good antimicrobial activity and resistance to gastrointestinal condition (1).

Multi-drug resistant bacteria are involved with several community acquired and nosocomial infections as well as foodborne diseases. Biofilm formation is regarded as a major determinant factor in development of infection by pathogenic bacteria (2). Biofilm is a community of microorganisms adhering to each other on a surface which is surrounded by a matrix of extracellular polymers. Bacteria embedded in a biofilm are more resistant to antimicrobials due to the reduction of antimicrobial penetration, slower bacterial metabolic state as well as easier exchange of resistance genes among cells (3). The resistant nature of biofilm is a matter of great concern for global health care system and many studies have been conducted to explore novel, natural and effective antibiofilm agents.
Fermented milk products contain different types of LAB which could be able to inhibit many pathogenic and spoilage microorganisms. Antagonism is attributed to the release of a variety of functionally active compounds including organic acids, exopolycacharides and bacteriocins which are naturally released into the bacterial growth medium. These compounds have antibacterial and antibiofilm potentials which are able to inhibit bacterial infectivity. Several studies have reported antibiofilm potential of bacteriocines produced by LAB bacteria (4-5). Moreover, some studies reported that a number of exopolysacharides isolated from commercial fermented milk were capable of interfering with the adhesion of several enteric pathogens (6-7).

The LAB from traditional cheese may produce bioactive compounds with antibiofilm activity and could be used for prevention of bacterial infections and food spoilage (4). However, the stability of these bioactive compounds under unfavorable conditions needs to be characterized. Thus, the current work was conducted to investigate and characterize antibiofilm potential of the cell free supernatant (CFS) of Lactobacillus plantarum spp, isolated from Siahmazgi cheese, a traditional cheese from province 
of Guilan, Iran, against multi-drug resistant Staphylococcus aureus, Escherichia coli and Pseudomonas aeroginusa, isolated from clinical infections.

\section{Materials and methods}

Microbial strains and culture conditions

Pathogenic bacteria including S. aureus, E. coli and $P$. aeroginusa were isolated from clinical specimens including urine, blood and respiratory secretions. Bacterial isolation was performed between February to June, 2017, from patients referring to the clinical laboratories of Rasht city, Iran. The patients had an age range of 3-68 year old. Bacterial identification was performed using biochemical assays. At first, Gram staining was performed and then, differential assays including coagulae, catalase, growth in manitole-salt agar and etc., for Gram positive isolates, and Motility, Indole and $\mathrm{H}_{2} \mathrm{~S}$ production, MR-VP, citrate utilization, sugar fermentation in TSI medium and oxidase assays, for Gram negative isolates were performed. All the isolates were stored at $-70{ }^{\circ} \mathrm{C}$ in Tryptic Soy broth (TSB) (QUELAB/UK) containing 20\% glycerol for subsequent analysis.

Antibiotic resistance profile of the isolates were determined using disk diffusion method and each strain which showed resistance to at least three antimicrobial classes was regarded as multi-drug resistant strain (8).

L. plantarum spp was isolated from Siahmazgi cheese and identified using biochemical and molecular assays. Briefly, cheese samples were collected aseptically, stored at $4{ }^{\circ} \mathrm{C}$ and transferred immediately to the laboratory. The samples were mixed and homogenized in $0.85 \%$ sterile $\mathrm{NaCl}$ solution $(1: 10 \mathrm{w} / \mathrm{v})$ on a shaker $(600 \mathrm{rpm})$ for ten minutes. Homogenized samples were diluted serially and cultured on MRS agar (Merck GmbH, Germany). After incubation at $37{ }^{\circ} \mathrm{C}$ for $48 \mathrm{~h}$, the Gram positive, Catalase negative, nonmotile rods were purified. Molecular identification of $L$. plantarum spp was performed by amplification and sequencing of the 16S rRNA gene of the isolate using the universal primers of 27 F: 5'AGAGTTTGATCATGGCTCAG-3' and 1492 R: 5'TACGGTTACCTTGTTACGACTT-3' (9). Then, $L$. plantarum spp was grown in MRS broth till stationary phase and bacterial cells were removed by centrifugation at $8000 \times \mathrm{g}$ for $15 \mathrm{~min}$. Then, the cell free supernatant was filtrated through a $0.45 \mu \mathrm{m}$ pore size syringe filter (Biofil/China) and stored at $4^{\circ} \mathrm{C}$ until use.

\section{In vitro biofilm assay}

Biofilm formation potential of microbial pathogen was investigated in 96-well micro-titer plates, according to the method described previously (10). Briefly, optical density of the isolated strains, grown in Luria Broth (LB) medium, was adjusted to 1.0 at $600 \mathrm{~nm}\left(\mathrm{OD}_{600}\right)$ and the cultures were diluted 1:100 in fresh LB. Then, $100 \mu \mathrm{L}$ of the diluted cultures were added to each well and the plates were incubated at $37^{\circ} \mathrm{C}$ for $48 \mathrm{~h}$ under static condition. Then, the plates were washed three times using sterile phosphate buffered saline (PBS) and $125 \mu \mathrm{L}$ of $0.1 \%$ crystal violet solution was added to the wells. After incubation for $15 \mathrm{~min}$ at room temperature the plates were washed and then left to dry. Finally, $125 \mu \mathrm{L}$ of $30 \%$ acetic acid was added to solubilize the dye and the $\mathrm{OD}_{550}$ was recorded using microplate reader (Bio-Rad, USA). The OD values were considered as biofilm formation level for each strain. Finally, ten isolates of each pathogen with the highest biofilm formation potential were used for antibiofilm assays.

\section{Anti-adhesive activity of the cell free supernatant}

Anti-adhesive potential of the CFS from L. plantarum spp against multi-drug resistant pathogens was investigated using the method reported by Dusane et al., (11). Briefly, bacterial pathogens were allowed to adhere for $4 \mathrm{~h}$ in microtiter plates and then were incubated with $100 \mu \mathrm{L}$ of the CFS from L. plantarum spp for $2 \mathrm{~h}$ at $37{ }^{\circ} \mathrm{C}$. After incubation, the wells were aspirated and washed with PBS and biofilm formation was quantified as described above.

\section{Disruption of preformed biofilms}

Biofilms of the pathogenic bacteria were allowed to form in micro-titer plates for $24 \mathrm{~h}$. Then, the medium was discarded from each well, rinsed with PBS and $100 \mu \mathrm{L}$ of the CFS was added to each well. The plates were incubated for a further $24 \mathrm{~h}$ at $37{ }^{\circ} \mathrm{C}$. After incubation, the medium was discarded and biofilm formation in each well was quantified using crystal violet method as described above (12).

Determination of physiochemical properties of $L$. plantarum CFS

In order to determine thermal stability, the CFSs was incubated at $25,37,50,75$ and $100{ }^{\circ} \mathrm{C}$ for $30 \mathrm{~min}$. In addition, stability of CFS at different $\mathrm{pH}$ was investigated in 
a range between 4 to 10 . Lactic acid was used to reduce the $\mathrm{pH}$ and a $1 \mathrm{M} \mathrm{NaoH}$ solution was used to increase the $\mathrm{pH}$. MRS broth with $\mathrm{pH}$ adjusted to different values were used as controls. In another experiment, CFS was prepared and treated with different chemicals $(10 \mathrm{mg} / \mathrm{mL})$ including Tween 80, SDS and EDTA for $30 \mathrm{~min}$ at $30{ }^{\circ} \mathrm{C}$. Finally, antibiofilm activity of the CFSs treated to different physicochemical conditions against clinical isolates of $P$. aeruginosa was determined and compared with controls.

Enzymatic characterization of antibiofilm potential of CFS In order to evaluate enzymatic susceptibility of antibiofilm compounds produced by L. plantarum, the CFS was treated with pepsin, proteinase $\mathrm{K}$ and lipase at the concentration of $0.5 \mathrm{mg} / \mathrm{mL}$. The tubes were incubated for $4 \mathrm{~h}$ at $37^{\circ} \mathrm{C}$ and then, antibiofilm activity of the CFSs against clinical isolates of $P$. aeruginosa were determined. MRS broth containing different enzymes with the mentioned concentration were used as controls.

\section{Statistical analysis}

The assays were performed in triplicates and the data were statistically evaluated using ANOVA and $\mathrm{p}$ values less than 0.05 were considered statistically significant (SPSS software, USA).

\section{Results}

\section{Biofilm inhibition of L. plantarum CFS}

Anti-adhesive potential of $L$. plantarum CFS against bacterial pathogens, which were able to produce strong biofilm, was investigated. According to the results, biofilm formation was significantly reduced when $P$. aeroginusa strains were treated with the CFS while the least antibiofilm activity was observed against $E$. coli strains. The attachment of $P$. aeroginusa strains to microtiter plates was significantly inhibited $(71-89 \%)$, with the mean value of $76.8 \%$. biofilm formation by $S$. aureus strains was inhibited in a range of 49-66\% (mean value $=58.6$ ) and biofilm inhibition of $52.2 \%$ was observed when $E$. coli strains were exposed to CFS. Biofilm inhibitory potential of the CFS against bacterial pathogens was presented in Fig 1 a and b.

\section{Disruption of preformed biofilms}

In our study, preformed biofilms of all bacterial pathogens were significantly disrupted by $L$. plantarum CFS $(P<$ 0.05), However, similar biofilm disruption levels was observed for all bacterial pathogens. Biofilms of $P$. aeroginusa, $S$. aureus and $E$. coli in microtiter plates were disrupted to $46.6 \%, 41.8 \%$ and $37.0 \%$, respectively (Fig. 2).
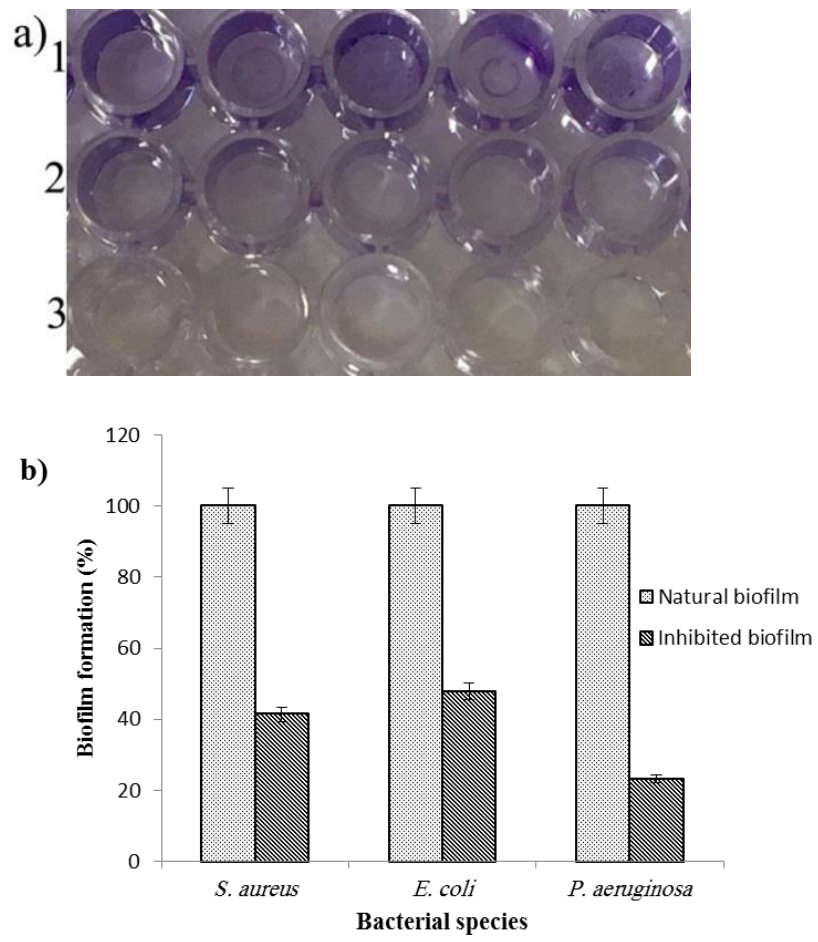

Figure 1 a) Biofilm inhibition by the CFS from $L$. plantarum spp. 1: natural biofilms produced by bacterial pathogens, 2: inhibited biofilms, 3: negative controls. b) Effect of the CFS from L. plantarum spp on biofilm formation of pathogenic bacteria.

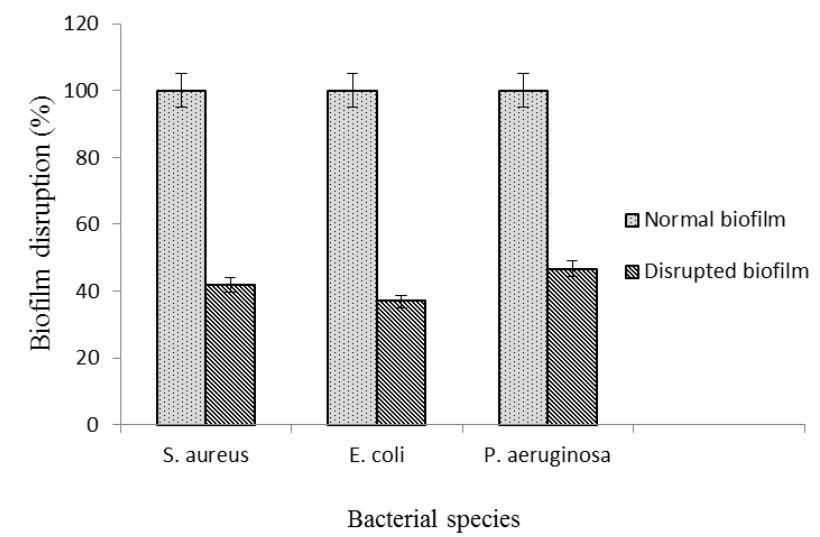

Figure 2 Disruption of preformed biofilms of bacterial pathogens by the CFS from $L$. plantarum spp.

ffect of different physiochemical conditions on antibiofilm activity of $\mathrm{CFS}$

According to the results, $L$. plantarum CFS remained stable after incubation at $30{ }^{\circ} \mathrm{C}$ at $\mathrm{pH} 4.0$ and 6.0. However, the antibiofilm activity partially reduced at $\mathrm{pH} 8.0$ and 10.0. In addition, incubation at different temperatures revealed heat stable nature of the CFS. Antibiofilm activity of the CFS against pathogenic bacteria did not reduce significantly when was incubated at $25,37,50$ and $75{ }^{\circ} \mathrm{C}$. However, a 
partial reduction of antibiofilm potential was observed when the CFS was incubated at $100{ }^{\circ} \mathrm{C}$ for $30 \mathrm{~min}$ (Fig 3 a and $\mathrm{b}$ ). Moreover, treating the CFS with different chemicals including SDS, tween 80 and EDTA did not significantly affect antibiofilm activity of the CFS.

a)
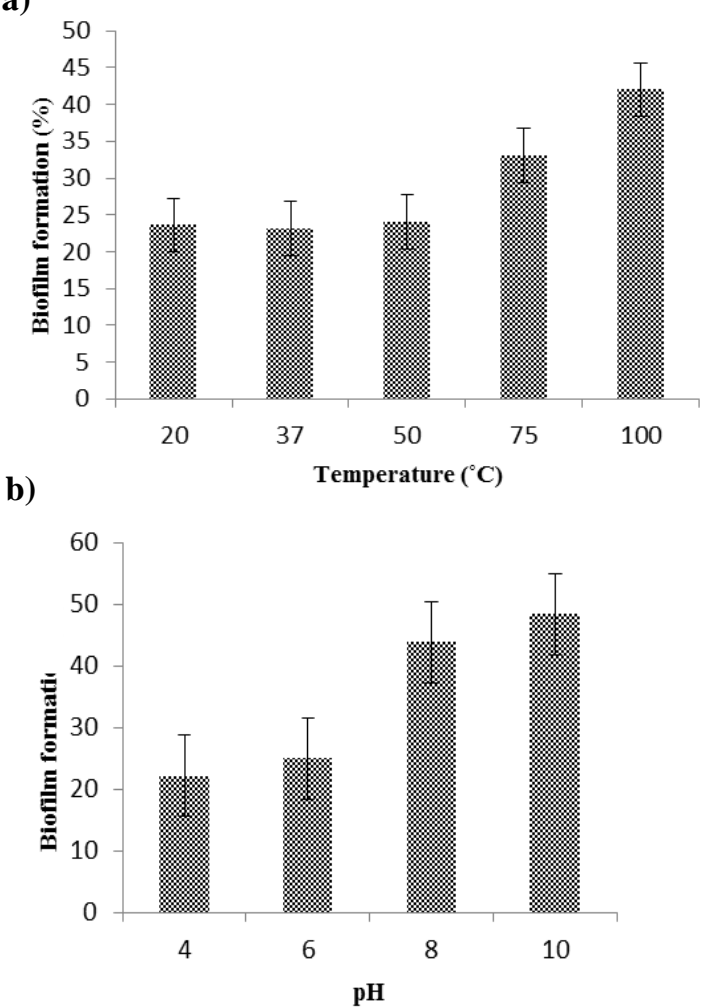

C)

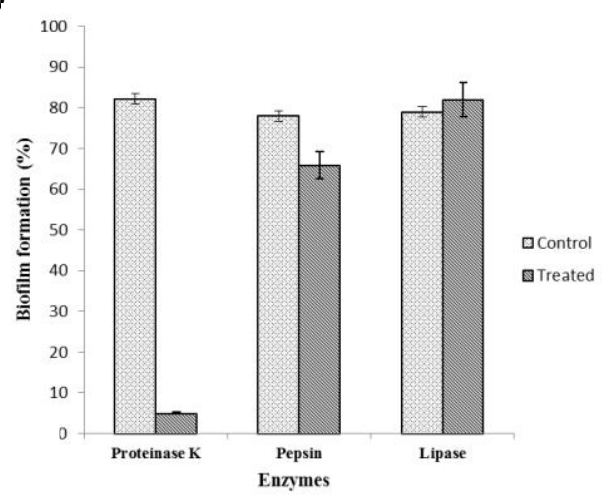

Figure 3 Effect of different physicochemical treatments on antibiofilm activity of CFS from $L$. plantarum spp against pathogenic $P$. aeruginosa strains. a) Temprature, b) $\mathrm{pH}$, c) Chemicals.

\section{Effect of different enzymes on antibiofilm activity of CFS} Incubation of $L$. plantarum CFS with proteinase $\mathrm{K}$ for $4 \mathrm{~h}$ at $37{ }^{\circ} \mathrm{C}$, totally inhibited antibiofilm potential of the $\mathrm{CFS}$, while incubation with pepsin partially reduced antibiofilm activity to $44.1 \%$, compared to the control. In addition, treating of the CFS with lipase insignificantly affected its antibiofilm activity (Fig 3c).

\section{Discussion}

In this study we isolated a $L$. plantarum spp from Siahmezgi cheese, a traditional cheese which is produced in the north of Iran, and determined its antibiofilm potential against multidrug resistance bacterial pathogens.

Occurrence of $L$. plantarum in cheese microbiota appears to be a common trait of a variety of milk cheeses (4). Isolation of L. plantarum strains from traditional cheese has been reported previously and have been shown to be able to produce a variety of antimicrobial compounds (13).

Ability to adhere to different surfaces and biofilm formation have been highlighted as important features associated to bacterial pathogens. In addition, biofilm formation is regarded as an important factor associated to the increased antibiotic resistance characteristic of bacterial pathogens. Traditional dairy products have been considered as good sources of $\mathrm{LAB}$ and ideal vehicles to deliver probiotic bacteria to human (1). LAB produce a variety of secondary metabolites which have antimicrobial and anticancer activities. The secondary metabolites are released in bacterial growth medium and so, the CFS could exhibit different biological activities. CFS from LAB contain a variety of biologically active compounds including exopolysaccharides and proteins. Our results showed CFS from L. plantarum spp strongly inhibited biofilm formed by all bacterial pathogens studied in this work. The highest antibiofilm activity was observed against $P$. aeruginosa, a bacterial pathogen which is involved with a variety of human infections. Several virulent factors are associated with bacterial initial adhesion to surfaces, which is followed by production of extracellular polymeric substances and result in biofilm formation. Antibiofilm activity of $L$. plantarum CFS could be associated to inhibition of both steps. Antibiofilm activity of the released exopolysaccharides derived from bacterial CFS has been reported, previously (6). Although, the molecular mechanism of biofilm inhibition by the CFS was not investigated in our work, it was possible that inhibition of biofilm was mediated by these exopolyshaccharised via modification of bacterial cell surfaces and thus prevention of initial attachment and/or acting as signal molecules which down-regulate expression of the genes involved with biofilm formation. Biofilm formation consists of initial bacterial adherence to the surface, via bacterial surface adhesions, followed by multiplication and production of extracellular polymeric matrix. Biofilm inhibitory potential of bacterial CFS could be associated to repression of the genes involved with initial adhesion and chemotaxis. Kim and Kim (6) reported that released exopolysaccharide (r- 
EPS) produced from probiotic bacteria reduced biofilm formation of enterohemorrhagic $E$. coli $\mathrm{O}_{157}: \mathrm{H}_{7}$ via repression of the curli production genes, including $\mathrm{crl}$, (2.0fold), $\operatorname{csg} A$ (1.8-fold), and $\operatorname{csg} B$ (1.7-fold,) and chemotaxis gene $c h e Y$ (2.2-fold). Inhibition of $c h e Y$ is also associated with biofilm inhibition in $P$. aeruginosa (14). In addition, functionally active compounds of bacterial CFS could act as signaling molecules which down-regulate expression of the biofilm modulating and/or quorum sensing genes (15). Biofilm formation is regulated by quorum sensing in a variety of bacterial species. Biofilm formation has been reported to be strongly affected by inhibition of quorum sensing signaling pathway in S. aureus (16). Thus, inhibition of biofilm in $S$. aureus by $L$. plantarum CFS could be associated with quorum sensing inhibition. However, further investigations needs to be performed to confirm this finding.

Bacterial pathogens exhibited different level of susceptibility to L. plantarum CFS. The difference could be associated to the difference in the cell surface characteristics, different signaling pathways as well as diversity of adhesions associated with each pathogen. Biofilm inhibitory potential of local isolates of LAB has been reported. Sadri et al., (17) reported a moderate antiobiofilm potential of CFS from $L$. casei and $L$. acidophilus which inhibited adhesion of uropathogenic $E$. coli strains by 46.7 and $25.3 \%$, respectively, which were less than antibiofilm activity of $L$. plantarum strain isolated in our study. The difference could be associated to the different characteristics of both LAB bacteria and microbial pathogens. In addition, the CFS from $L$. casei showed strong biofilm inhibitory potential against $P$. aeruginosa (18) (up to 87\%) which was in accordance to our results. Aminnezhad and Kasra-Kermanshahi (18) associated antibiofilm activity of the CFS to the modulation of quorum sensing signaling pathway of bacterial pathogen.

Disruption of preformed biofilm by L. plantarum CFS was also investigated. Our results showed that the CFS disrupted bacterial biofilms efficiently. However, there was not a significant difference in the biofilm disruption level among the bacterial pathogens. Disruption of the preformed biofilms are involved with digestion of the extracellular compounds which are produced by biofilm forming cells which cause cell aggregation. It seems that several digestive enzymes are released in the CFS and could be associated with disruption of the preformed biofilm. Similar levels of biofilm disruption against pathogenic bacteria by $L$. plantarum CFS could be associated to the similar nature of polymeric matrix produced by bacterial pathogens. In addition, lower antibiofilm activity of $L$. plantarum CFS against preformed biofilms compared to the free cells, is evidence that cells in a biofilm are more resistant to antimicrobial agents compared to free-floating cells (19). Physicochemical characterization of L. plantarum CFS at different conditions, showed the CFS is stable at a wide range of temperatures and $\mathrm{pH}$. However, the CFS showed better antibiofilm potential at low $\mathrm{pH}$. Accordingly, we suppose the higher antibiofilm activity at low $\mathrm{pH}$ could be attributed to the higher solubility of antibiofilm compounds, better folding of proteins and/or enhanced binding and blocking of adhesions of bacterial pathogens. Higher antimicrobial activity of $L$. plantarum CFS at low $\mathrm{pH}$ has been reported previously by several authors (20-21). In addition, antibiofilm potential of $L$. plantarum CFS did not significantly reduced when was exposed to high temperatures. Heat stability of the CFS is a good feature which could be used in food industries.

Antibiofilm activity of the CFS showed good stability in presence of different chemicals including EDTA, SDS and tween 80. High stability of the CFS in presence of the mentioned chemicals indicates its stable antiobiofilm potential to be used as biopreservatives and pharmaceutical compounds.

Antibiofilm potential of $L$. plantarum CFS was completely inhibited by Proteinase K. This finding has been reported previously for bacteriocines produced by $L$. plantarum, indication the role of bacteriocines in biofilm inhibition of the CFS from L. plantarum spp (22). In addition, antibiofilm activity of the CFS was not strongly affected by the lipase which shows the antibiofilm activity is not dependent on the lipid compounds of the CFS.

\section{Conclusion}

In this study a L. plantarum spp was isolated from traditional cheese of province of Guilan and antibiofilm potential of the L. plantarum CFS against clinical isolates of $P$. aeruginosa, $S$. aureus and $E$. coli was investigated. This work showed that traditional dairy products are good sources of beneficial bacterial strains and bioactive compound released by these strains could be employed in food and pharmaceutical industries for prevention of bacterial infections and contamination. However, purification and identification of the functionally active compounds with antibiofilm activity from $L$. plantarum CFS, characterization their functionality at invivo condition and evaluation of the exact molecular mechanisms involved with biofilm inhibition, were the limitations of this study which need to be focused in future works. 


\section{Acknowledgments}

The authors would like to thank the University of Guilan for providing facilities to carry out this work. This work was supported by University of Guilan (grant number 1222).

\section{Conflict of interest}

The authors declare that there is no conflict of interest.

\section{Authors' contribution}

Study design and data interpretation was performed by Dr. H. Zamani and all authors were involved with the experimental assays, data acquisition and drafting of the manuscript.

\section{References}

1. Zamani $\mathbf{H}$. Isolation of a potentially probiotic Lactobacillus plantarum from Siahmezgi cheese and its characterization as a potentially probiotic. Biol J Microorgan 2016;4:97-108.

2. Salehzadeh A, Zamani H, Langeroudi MK, Mirzaie A. Molecular typing of nosocomial Staphylococcus aureus strains associated to biofilm based on the coagulase and protein A gene polymorphisms. Iran J Basic Med Sci 2016;19:1325-30.

3. Khoramian B, Jabalameli F, Niasari-Naslaji A, Taherikalani M, Emaneini M. Comparison of virulence factors and biofilm formation among Staphylococcus aureus strains isolated from human and bovine infections. Microb Pathog 2015;88:73-7.

4. Milioni C, Martínez B, Degl'Innocenti S, Turchi B, Fratini F, Cerri D. and Fischetti R. A novel bacteriocin produced by Lactobacillus plantarum LpU4 as a valuable candidate for biopreservation in artisanal raw milk cheese. Dairy Sci Tech 2015; 95:479-94.

5. Martinez RC, Wachsman M, Torres NI, LeBlanc JG, Todorov SD, Franco BD. Biochemical, antimicrobial and molecular characterization of a noncytotoxic bacteriocin produced by Lactobacillus plantarum ST71KS. Food Microbiol 2013;34:376-81.

6. Kim Y, Kim SH. Released exopolysaccharide (r-EPS) produced from probiotic bacteria reduce biofilm formation of enterohemorrhagic Escherichia coli O157: H7. Biochem Biophys Res Commun 2009; 379: 324-9.

7. Ruas-Madiedo P, Gueimonde M, De Los Reyes-Gavilan CG, Salminen S. Effect of exopolysaccharide isolated from "viili" on the adhesion of probiotics and pathogens to intestinal mucus. J Dairy Sci 2006;89: 2355-8.

8. CLSI. Performance Standards for Antimicrobial Susceptibility Testing; Twenty-Fifth Informational Supplement. CLSI Document M100-S25, Clinical and Laboratory Standards Institute 2015;1-76.
9. Lillo AD, Ashley FP, Palmer RM, Munson MA, Kyriacou L, Weightman AJ, et al. Novel subgingival bacterial phylotypes detected using multiple universal polymerase chain reaction primer sets. Molecul Oral Microbiol 2006;21:61-8.

10. Das S, Dash HR. Microbial Biotechnology- A laboratory Manual for Bacterial Systems. Springer India. 2015.

11. Dusane DH, Pawar VS, Nancharaiah YV, Venugopalan VP, Kumar AR, Zinjarde SS. Anti-biofilm potential of a glycolipid surfactant produced by a tropical marine strain of Serratia marcescens. Biofouling 2011;27:645-54

12. Dusane DH, Rajput JK, Kumar AR, Nancharaiah YV, Venugopalan VP, Zinjarde SS. Disruption of fungal and bacterial biofilms by lauroyl glucose. Lett Appl Microbiol 2008; 47:374-9.

13. Lavilla-Lerma L, Perez-Pulido R, Martinez-Bueno M, Maqueda M, Valdivia E. Characterization of functional, safety, and gut survival related characteristics of Lactobacillus strains isolated from farmhouse goat's milk cheeses. Int J Food Microbiol 2013;163:136-45.

14. Barken KB, Pamp SJ, Yang L, Gjermansen M, Bertrand JJ, Klausen M, et al. Roles of type IV pili, flagellum-mediated motility and extracellular DNA in the formation of mature multicellular structures in Pseudomonas aeruginosa biofilms. Environ Microbiol 2008;10:233143.

15. Wang J, Zhao X, Yang Y, Zhao A, Yang Z. Characterization and bioactivities of an exopolysaccharide produced by Lactobacillus plantarum YW32. Int J biol macromol 2015;74:119-26.

16. Balaban N, Cirioni O, Giacometti A, Ghiselli R, Braunstein JB, Silvestri $\mathrm{C}$, et al. Treatment of Staphylococcus aureus biofilm infection by the quorum-sensing inhibitor RIP. Antimicrob agents chemother 2007;51:2226-9.

17. Sadri M, Arab Soleimani N, Forghanifard MM. The study of Antimicrobial and Anti-adhesive effect of ProbioticLactobacilli on Uropathogenic Escherichia coli (UPEC). Biol J Micoorgan; 2016;5:159-70.

18. Aminnezhad S, Kasra-Kermanshahi R. Antibiofilm activity of cell-free supernatant from Lactobacillus casei in Pseudomonas aeruginosa. Feyz 2014;18:30-7.

19. Krysinski EP, Brown LJ, Marchisello TJ. Effect of cleaners and sanitizers on Listeria monocytogenes attached to product contact surfaces. J Food Prot 1992;55:246-51.

20. Hu M, Zhao H, Zhang C, Yu J, Lu Z. Purification and characterization of plantaricin 163, a novel bacteriocin produced by Lactobacillus plantarum 163 isolated from traditional Chinese fermented vegetables. J Agric Food Chem 2013;61:11676-82.

21. Zhu X, Zhao Y, Sun Y, Gu Q. Purification and characterisation of plantaricin ZJ008, a novel bacteriocin against Staphylococcus spp. from Lactobacillus plantarum ZJ008. Food Chem 2014;165:216-23.

22. Kelly WJ, Asmundson RV, Huang CM. Isolation and characterization of bacteriocin-producing lactic acid bacteria from ready-to-eat food products. Int J F Microbiol 1996;33:209-18 